\title{
Density functional study of carbonic acid clusters
}

\author{
P. Ballone, ${ }^{a)}$ B. Montanari, ${ }^{\text {b) }}$ and R. O. Jones ${ }^{\mathrm{c})}$ \\ Institut für Festkörperforschung, Forschungszentrum Jülich, D-52425 Jülich, Germany
}

(Received 24 November 1999; accepted 26 January 2000)

\begin{abstract}
Density functional calculations on carbonic acid $\mathrm{H}_{2} \mathrm{CO}_{3}$ are extended to clusters of up to five such units. The most stable forms are the linear, hydrogen-bonded analogs of the dimer with anti-anti orientation. We calculate structures and vibration frequencies, as well as the energy required to bend and stretch the linear isomers. Linear chains of up to $\sim 20$ units should be favored over ring structures, and they have a tensile strength reminiscent of chains of water molecules. We also discuss planar, nonlinear structures as well as three-dimensional isomers. (C) 2000 American Institute of Physics. [S0021-9606(00)30615-8]
\end{abstract}

\section{INTRODUCTION}

The interaction of carbon dioxide with water plays a central role in many biological, ecological, extraterrestrial (particularly cometary), and industrial processes. Carbonic acid $\left(\mathrm{H}_{2} \mathrm{CO}_{3}\right.$, denoted $\left.\mathrm{CA}\right)$, formed by the hydration of $\mathrm{CO}_{2}$, dissociates rapidly in water, so that the evidence for its existence is often indirect. There have been numerous calculations of the energetics of the gas phase reaction: ${ }^{1,2}$

$$
\mathrm{H}_{2} \mathrm{O}(g)+\mathrm{CO}_{2}(g) \rightleftharpoons \mathrm{H}_{2} \mathrm{CO}_{3}(g) .
$$

The most extensive calculations indicate that there is an activation barrier for reaction (1) of over $50 \mathrm{kcal} / \mathrm{mol}^{1,3}$ although it is catalyzed by the presence of additional water molecules. ${ }^{3}$ A peak with mass-to-charge ratio of 62 following thermal decomposition of $\left(\mathrm{NH}_{4}\right) \mathrm{HCO}_{3}$ (s) has been attributed to gas phase carbonic acid, ${ }^{4}$ and other weakly bound complexes - such as $\mathrm{H}_{2} \mathrm{O}-\mathrm{CO}_{2}$ - have been found in the gas phase. $^{5}$

Low temperatures would favor the formation of carbonic acid, and two techniques have been used to synthesize $\mathrm{H}_{2} \mathrm{CO}_{3}$ at low $T$ and to detect it using infrared (IR) spectroscopy. Moore and Khanna ${ }^{6}$ performed proton irradiation on mixed $\mathrm{H}_{2} \mathrm{O}$ and $\mathrm{CO}_{2}$ ices at $20 \mathrm{~K}$, and carried out IR and mass spectroscopic studies on the products. New features in the IR spectra of the resulting films were assigned to solid $\mathrm{H}_{2} \mathrm{CO}_{3}$ trapped in the ice matrix. Hage et al. ${ }^{7,8}$ formed solid $\mathrm{H}_{2} \mathrm{CO}_{3}$ by the reaction of $\mathrm{K}_{2} \mathrm{CO}_{3}$ and $\mathrm{HCl}$ (and $\mathrm{HBr}$ ) at 150 $\mathrm{K}$ in vitreous methanol films. Crystallization to a form denoted $\alpha-\mathrm{H}_{2} \mathrm{CO}_{3}$ occurred at $190-200 \mathrm{~K}$, and Fourier transform infrared spectroscopy (FTIR) measurements were performed on it. When water was used as solvent, a second metastable polymorph $\left(\beta-\mathrm{H}_{2} \mathrm{CO}_{3}\right)$ could be identified.

The small energy differences involved in the formation of carbonic acid and the metastable nature of many of the structures involved provide challenges to methods for mo-

\footnotetext{
a)Permanent address: Università degli Studi di Messina, Dipartimento di Fisica, Contrada Papardo, I-98166 Messina, Italy.

b) Present address: Department of Physics, Queen's University, Belfast BT7 1NN, Northern Ireland.

c) Author to whom correspondence should be addressed. Electronic mail: r.jones@fz-juelich.de
}

lecular structure calculations. The $\mathrm{H}_{2} \mathrm{CO}_{3}$ dimer is a particularly interesting case, as very extensive calculations ${ }^{2}$ indicate that this cluster is remarkably stable. The energy difference between the dimer and the constituent $\mathrm{CO}_{2}$ and $\mathrm{H}_{2} \mathrm{O}$ molecules is, when corrected for zero point energy differences, "astonishingly close to zero." 2 The dimer is, therefore, more relevant for investigating the stability of $\mathrm{H}_{2} \mathrm{CO}_{3}$ than is the monomer. The stability of still larger clusters is a tantalizing question that, to the best of our knowledge, has not yet been addressed.

In an earlier paper, ${ }^{9}$ we discussed the bonding and energy differences of numerous fragments of bisphenol-A polycarbonate (BPA-PC), including the isomers of $\mathrm{H}_{2} \mathrm{CO}_{3}$ shown in Fig. 1. The most stable is the trans-trans isomer, followed by the cis-trans and cis-cis forms. The calculated frequencies are in reasonable agreement with other calculations and with experimental data. In the present work, we extend these calculations to small aggregates of $\mathrm{H}_{2} \mathrm{CO}_{3}$ molecules. It continues a study of the applicability of the density functional (DF) method to organic molecules and molecular crystals, as well as to organic polymers. In addition to the intrinsic interest in aggregates of hydrogen-bonded molecules, it is a challenge to theory to provide insight into the presently unknown structures of the carbonic acid polymorphs. Essential details of the calculations are provided in Sec. II, and the results for the structures and vibration frequencies are given in Sec. III. We discuss and summarize our findings in Sec. IV.

\section{METHODS OF CALCULATION}

The method of calculation has been described previously. ${ }^{10,11}$ The electron-ion interaction is represented by ionic pseudopotentials with the (nonlocal) form suggested by Troullier and Martins. ${ }^{12}$ We adopt periodic boundary conditions with a simple cubic cell with lattice constant 36 a.u. and a plane wave basis with a kinetic energy cutoff of 35 a.u., and the expansion of the orbitals uses a single point $(\mathbf{k}=0)$ in the Brillouin zone. As in previous work in this series, we use the exchange-correlation energy functional of Perdew, Burke, and Ernzerhof (PBE) ${ }^{13}$ which is relatively simple and free of empirical input. All structures were opti- 


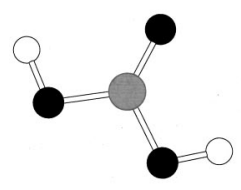

(a)

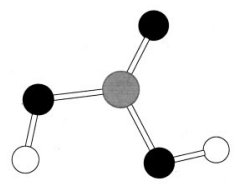

(b)

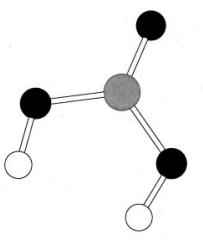

(c)

FIG. 1. Molecular structures. (a) trans-trans (b) cis-trans, and (c) cis-cis isomers of carbonic acid. Carbon atoms are gray, oxygen atoms black, hydrogen atoms white.

mized using a combination of DF calculations with molecular dynamics (MD) $)^{14,15}$ and a simulated annealing strategy. Optimizations were continued until the magnitude of the maximum force on any atom was less than $5 \times 10^{-4}$ a.u. and the average force on the atoms are an order of magnitude less.

The small energy differences involved in hydrogen bonding have provided a challenge for all methods of molecular structure calculations. Tests on the prototype water dimer $\left(\mathrm{H}_{2} \mathrm{O}\right)_{2}$ showed that the local density (LD) approximation to the exchange-correlation energy overbind this system, while both Hartree-Fock and many-body perturbation theory (MBPT) lead to significant underbinding. ${ }^{16}$ Gradient corrections to the LD functional lead to dramatic improvements and good agreement with measured structures and vibration frequencies, and these corrections have subsequently been used in numerous studies of hydrogen-bonded systems. ${ }^{17}$ In particular, a thorough study of the Ih phase of ice found that the PBE functional gave excellent results for the sublimation energy, equilibrium volume, and bulk modulus. ${ }^{18}$ Finally, a recent study showed that this functional provides a very satisfactory description of atomization energies for the extended G2 set of molecules. ${ }^{19}$ All this experience with the PBE functional is very encouraging for the present study of carbonic acid clusters.

The large computational demands of this method mean that it does not suffice to generate starting configurations in organic and other molecules with strong, directional bonds if the structures are unknown. To accelerate the exploration of the potential energy surface, we have used classical molecular dynamics with an empirical force field. ${ }^{20}$ This allows us to perform extensive simulated annealing on many structures and has provided input to DF calculations of structures that we had not found with other means. Nevertheless, we have concluded that the empirical potential is of limited use for $\mathrm{CA}_{n}$ clusters, since it is difficult to incorporate simply the pronounced tendency to planarity displayed by adjacent hydrogen bonds. As a result, many low-energy structures generated by this method were found to be unstable at the level of DF calculations.

\section{RESULTS}

\section{A. Monomers, dimer}

In Fig. 1 we show the structures of the (a) trans-trans, (b) cis-trans, and (c) cis-cis isomers of $\mathrm{H}_{2} \mathrm{CO}_{3}$, which we discussed in Ref. 9. For completeness we provide (Table I) the structural parameters and energy differences calculated using the present method, as well as the results of other calculations. ${ }^{1,21}$

The small energy differences involved in the reaction of water and carbon dioxide mean that vibrational zero point energy (ZPE) corrections are important. ${ }^{2}$ Our calculations indicate that the reaction $\mathrm{H}_{2} \mathrm{CO}_{3} \rightarrow \mathrm{H}_{2} \mathrm{O}+\mathrm{CO}_{2}$ at $T=0 \mathrm{~K}$ re-

TABLE I. Bond lengths $(\AA)$ and angles (degrees), and differences in total and zero point energy $\left(\Delta E, \Delta E_{Z P}\right.$, $\mathrm{kcal} / \mathrm{mol}$ ) in carbonic acid isomers. The carbonyl group is $\mathrm{C} 7-\mathrm{O} 19$, and $\mathrm{H} 17$ and $\mathrm{H} 18$ are attached to $\mathrm{O} 17$ and O18, respectively (see Ref. 9). Values in parentheses are HF/6-31G* (Ref. 21) and MP-2 results (Ref. 1), respectively.

\begin{tabular}{lccc}
\hline \hline Struct. param. & trans-trans & cis-trans & cis-cis \\
\hline C7-O19 & $1.217(1.188,1.218)$ & $1.208(1.180,1.208)$ & $1.202(1.172,1.201)$ \\
C7-O17 & $1.353(1.315,1.345)$ & $1.371(1.316,1.344)$ & $1.371(1.333,1.366)$ \\
C7-O18 & $1.353(1.315,1.345)$ & $1.351(1.333,1.364)$ & $1.371(1.333,1.366)$ \\
O17-H17 & $0.984(0.951,0.969)$ & $0.985(0.950,0.969)$ & $0.982(0.947,0.966)$ \\
O18-H18 & $0.984(0.951,0.969)$ & $0.985(0.952,0.969)$ & $0.982(0.947,0.966)$ \\
O19-C7-O17 & $125.8(125.1,125.9)$ & $125.4(124.4,125.4)$ & $121.6(122.1,122.4)$ \\
O19-C7-O18 & $125.8(125.1,125.9)$ & $124.1(124.6,124.3)$ & $121.6(122.1,122.4)$ \\
H17-O17-C7 & $105.0(107.9,106.1)$ & $105.6(108.3,106.7)$ & $112.5(115.0,111.9)$ \\
H17 $\cdots$ O19 & $2.320(2.291, \ldots)$ & $2.336(2.299, \ldots)$ & $3.062(2.996, \ldots)$ \\
H18 $\cdots$ O19 & $2.320(2.291, \ldots)$ & $3.036(2.975, \ldots)$ & $3.062(2.996, \ldots)$ \\
H18 $\cdots$ O17 & $3.022(2.966, \ldots)$ & $2.170(2.993, \ldots)$ & $2.385(3.340, \ldots)$ \\
H17 $\cdots$ O18 & $3.022(2.966, \ldots)$ & $3.057(2.159, \ldots)$ & $2.385(3.340, \ldots)$ \\
H17 $\cdots$ H18 & $3.720(3.670, \ldots)$ & $3.134(3.096, \ldots)$ & $2.024(2.027, \ldots)$ \\
$\Delta E$ & 0.00 & $1.50(2.44,1.86)$ & $10.0(12.29,11.76)$ \\
$\Delta E_{Z P}$ & 0.00 & $-0.49(-0.46,-0.38)$ & $-1.78,(-1.90, \ldots)$ \\
\hline \hline
\end{tabular}




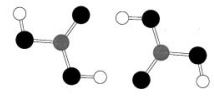

(a)

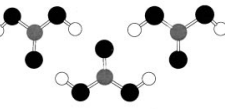

(b)

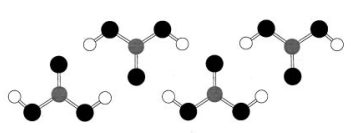

(c)

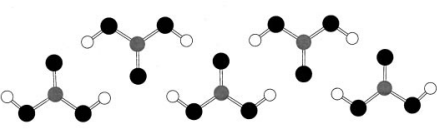

(d)

FIG. 2. Structures of planar carbonic acid clusters: (a) dimer, (b) trimer, (c) tetramer, (d) pentamer. Carbon atoms are gray, oxygen atoms black, hydrogen atoms white.

leases $7.1 \mathrm{kcal} / \mathrm{mol}$, with contributions from the potential energy and ZPE of $2.8 \mathrm{kcal} / \mathrm{mol}$ and $4.3 \mathrm{kcal} / \mathrm{mol}$, respectively. These results are in fair agreement with those of Ref. 1 , but they agree remarkably well with the most extensive calculations performed to date $[\operatorname{CCSD}(\mathrm{T})]^{2}$

A pair of CA molecules can decrease their energy by forming two equivalent hydrogen bonds, as illustrated in Fig. 2(a). The dimer is planar and has a negligible dipole moment, and the structural parameters have standard values $\left(d_{\mathrm{O}-\mathrm{O}}=2.57 \AA, \alpha_{\mathrm{O}-\mathrm{H}-\mathrm{O}}=178^{\circ}\right)$. The binding energy per bond $(10.3 \mathrm{kcal} / \mathrm{mol})$ is in good agreement with previous estimates using correlated wave functions (MP2, 9.5 $\mathrm{kcal} / \mathrm{mol})^{2}$ and a different gradient-corrected functional (B3LYP, $8.9 \mathrm{kcal} / \mathrm{mol}){ }^{2}$ The strength of the two hydrogen bonds is reflected in small but significant changes in the structure of the constituent monomers: the $\mathrm{C}=\mathrm{O}$ bond increases from $1.22 \AA$ in the monomer to $1.25 \AA$ in the dimer, the $\mathrm{C}-\mathrm{O}$ bond shrinks from $1.35 \AA$ to $1.32 \AA$, and the bond angles change slightly to adapt to the formation of the hydrogen bonds. The "external" $\mathrm{C}-\mathrm{O}-\mathrm{H}$ groups have almost identical geometries in monomer and dimer.

Hydrogen-bond formation in the dimer is also reflected in the vibrational frequencies, which display large redshifts from the corresponding modes for the isolated $\mathrm{H}_{2} \mathrm{CO}_{3}$ molecule: the stretching frequency of the $\mathrm{O}-\mathrm{H}$ groups involved in the intermolecular bonding decreases by more than $800 \mathrm{~cm}^{-1}$, and the $\mathrm{C}=\mathrm{O}$ stretching mode softens by more than $60 \mathrm{~cm}^{-1}$. The strong coupling of these modes gives rise to a large splitting for the $\mathrm{O}-\mathrm{H}\left(\Delta \omega=140 \mathrm{~cm}^{-1}\right)$ and the $\mathrm{C}=\mathrm{O}\left(\Delta \omega=100 \mathrm{~cm}^{-1}\right)$ stretching modes. The pronounced tendency of the dimer towards planarity is evident in the fact that the highest frequency out-of-plane mode $\left(1100 \mathrm{~cm}^{-1}\right)$ is associated with the torsion of the $\mathrm{OH}$ groups forming hydrogen bonds, while the corresponding modes in the isolated $\mathrm{H}_{2} \mathrm{CO}_{3}$ molecules occur at much lower frequency $\left(640 \mathrm{~cm}^{-1}\right)$. The combined effect of these changes in the vibrational spectrum is a slight destabilization (by $1 \mathrm{kcal} / \mathrm{mol}$ per monomer) of the dimer with respect to two separated monomers.

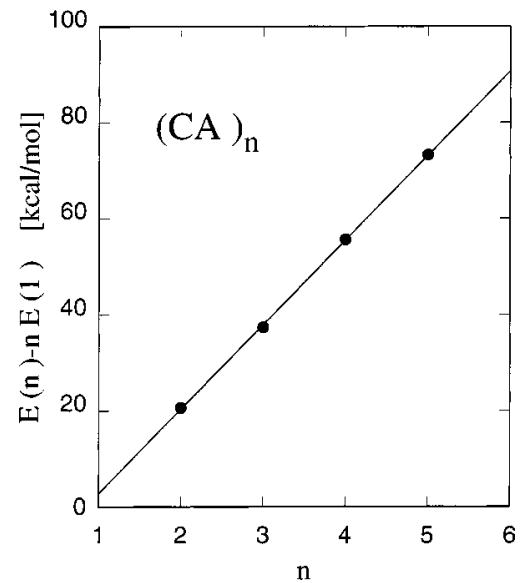

FIG. 3. Cohesive energy of linear clusters of carbonic acid $\mathrm{CA}_{n}$ as a function of $n$.

\section{B. Linear structures of trimer, tetramer, and pentamer}

The regular shape of the dimer suggests an obvious pattern for the growth of $\mathrm{CA}_{n}$ aggregates, as illustrated in Figs. 2(b)-2(e). Our calculations on the ladder structures up to $n$ $=5$ show that they are very stable, with cohesive energies growing linearly with $n$ (see Fig. 3). Each structure exhibits two sets of hydrogen bonds: the $\mathrm{C}=\mathrm{O}$ groups in the terminal molecules are involved in a single hydrogen bond, while those in the internal molecules of the ladder participate in two hydrogen bonds (see Fig. 2). As expected, the terminal bonds are slightly stronger than the internal ones, and the cohesive energy $E_{n}$ of the $\mathrm{CA}_{n}$ molecule can be represented by $E_{n}=20.6+17.0(n-2) \mathrm{kcal} / \mathrm{mol} .^{22}$ In Fig. 3 we show that this linear relation provides a very accurate fit to the calculated cohesive energies, and it can be used to predict the properties of longer linear CA structures. The structural trends observed in the dimer are also present in the larger species, with changes due to the presence of the two hydrogen-bond types described above: The internal $\mathrm{C}=\mathrm{O}$ and $\mathrm{C}=\mathrm{O}$ bonds $\left(d_{\mathrm{C}=\mathrm{O}}=1.27 \AA, d_{\mathrm{C}-\mathrm{O}}=1.31 \AA\right)$ expand and shrink, respectively, while the external hydrogen bonds and the extremal $\mathrm{C}-\mathrm{O}-\mathrm{H}$ groups are the same as in the dimer. The dipole moments of the clusters are very close to zero for even values of $n$ and are small for odd values. ${ }^{23}$

The evolution of the vibrational spectrum with increasing $n$ is shown in Fig. 4. The formation and the growth of the band representing the stretching of the $\mathrm{O}-\mathrm{H}$ groups involved in an intramolecular hydrogen bond is evident in the $2400-3000 \mathrm{~cm}^{-1}$ frequency interval, as is the progressive hybridization of the $\mathrm{C}=\mathrm{O}$ stretching modes $\left(1780 \mathrm{~cm}^{-1}\right.$ in the isolated monomer) with the bending modes of the $\mathrm{OH}$ groups (in the $1200-1400 \mathrm{~cm}^{-1}$ range in the monomer). The highest frequency band (at $3530 \mathrm{~cm}^{-1}$ ) is associated with the extremal $\mathrm{OH}$ groups. It is not affected by the formation of the intermolecular hydrogen bonds and changes little with increasing size. We note that the calculated vibration frequencies are generally consistent with the infrared data of the solid phases, ${ }^{6,7}$ but a detailed comparison between theory and experiment is made difficult by the lack of structural information in the latter and error bars of $\sim 30 \mathrm{~cm}^{-1}$ in the former. 


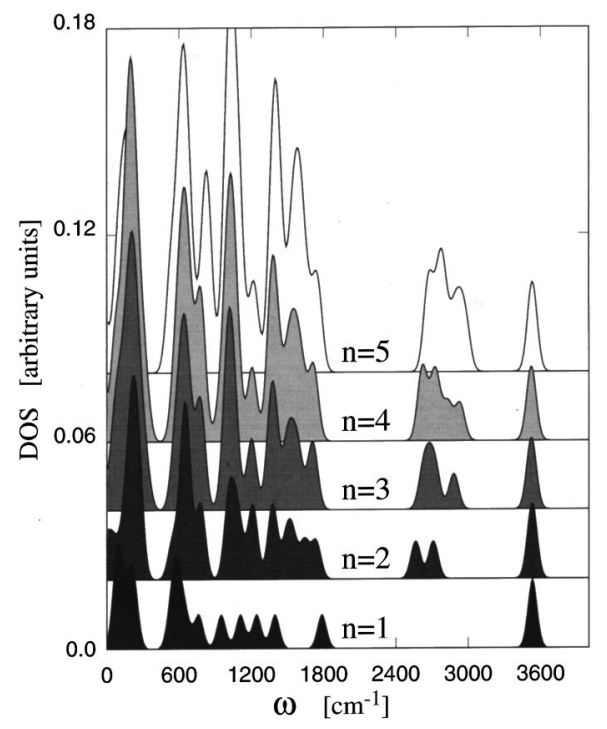

FIG. 4. Vibrational densities of states of linear clusters of carbonic acid $\mathrm{CA}_{n}$ for $n=1-5$. The states are broadened with a Gaussian of width $20 \mathrm{~cm}^{-1}$.

Linear structures are favored for clusters of some elements up to a certain size, beyond which the additional bond formed in a ring structure overcomes the energy cost of bending. An example is provided by carbon clusters $\mathrm{C}_{n}$, where this transformation takes place at $n \sim 8 .^{24}$ The same effect should be evident in $\mathrm{CA}_{n}$ clusters, where an energy lowering of $\sim 20 \mathrm{kcal} / \mathrm{mol}$ should result from the saturation of the external hydrogen-bond sites. The size of the ring where the gain in potential energy overcomes the cost of bending the linear geometries can be estimated by considering the pentamer and constraining the carbonyl groups of the central and extremal CA molecules to lie on a circle of radius $R$. The energy increase with respect to the linear geometry has been computed by relaxing all other degrees of freedom. The result, shown in Fig. 5 as a function of $1 / R^{2}$, indicates that the stiffness of the linear structure is such that ring formation is unfavorable up to $n \sim 20$. Entropy considerations render unlikely the formation of rings of this size.

Hydrogen bonding is often cited as the source of the tensile strength of water that helps plants transport liquids from the roots to the topmost branches and leaves..$^{25}$ To study whether linear clusters of CA display an analogous mechanical strength, we have evaluated the energy of a tetramer $\mathrm{CA}_{4}$ by fixing the distance between the outermost car-

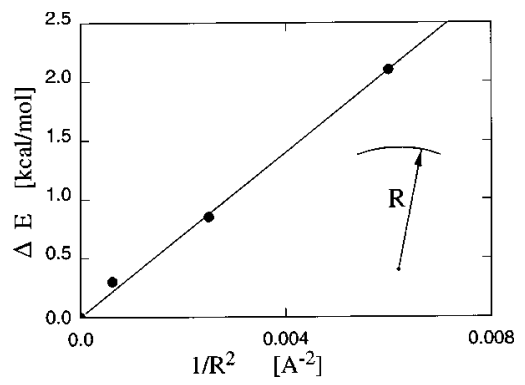

FIG. 5. Energy change on bending $\mathrm{CA}_{5}$ as a function of $1 / R^{2}$, where $R$ is the radius of curvature of the structure (see text).

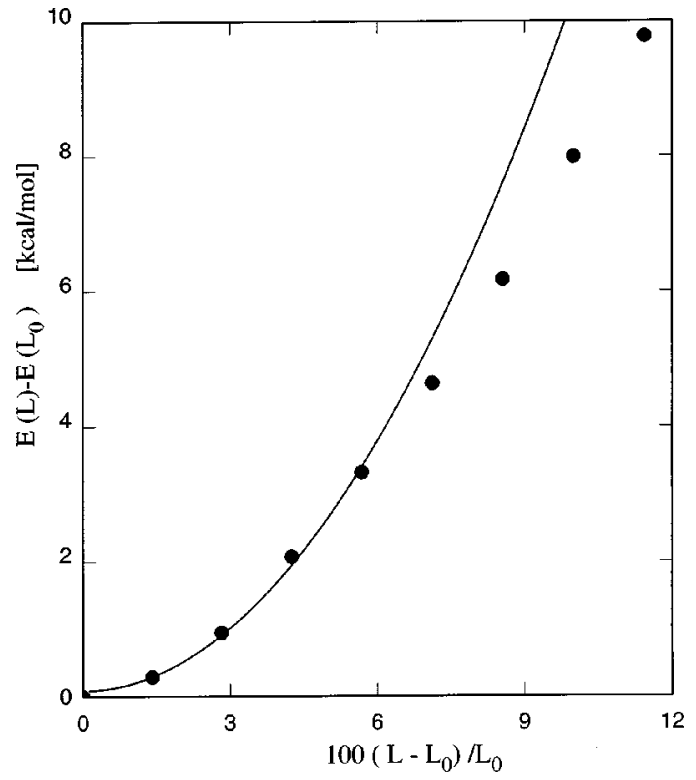

FIG. 6. Energy change in $\mathrm{CA}_{4}$ on increasing the separation $L$ between the outermost carbonyl groups (see text). $L_{0}$ is the equilibrium value of $L$.

bonyl groups to be larger than the equilibrium value, and relaxing all other degrees of freedom. Figure 6 shows that the deformation of the cluster is remarkably elastic up to $\sim 6 \%$ of the original length. The curvature of the elastic part leads to an estimate of the molecular elastic modulus (1.4 $\times 10^{11} \mathrm{~N} / \mathrm{m}^{2}$ ) that is similar to that found in well-annealed ice samples. ${ }^{26,27}$

\section{Nonlinear isomers}

Two crystalline forms of carbonic acid have been identified, ${ }^{6-8}$ and it is natural to ask whether threedimensional structures can be derived from CA clusters. Linear chains of CA can clearly give rise to extended solids bound by van der Waals interactions, but the absence of structural information suggests that we also investigate nonlinear isomers of CA clusters, even if they are less stable than their linear counterparts. The systematic search for isomers is notoriously difficult, and we have noted that the use of a simple interatomic potential (see Sec. II) is of limited value: the large gain in the speed of searching configuration space is offset by the fact that many isomers are unstable or very high in energy when studied with DF calculations. Without making any claim to completeness, we now present several examples of these isomers.

A nonlinear, planar isomer of $\mathrm{CA}_{3}$ is illustrated in Fig. 7(a). It has a single hydrogen bond per monomer, and it is not surprising that it is only marginally stable with respect to dissociation into the dimer and an isolated monomer (the binding energy with respect to three monomers is $24.3 \mathrm{kcal} /$ mol). On the other hand, this isomer has three sites for binding an additional monomer, and we show a typical planar structure built on it in Fig. 7(b). Unfortunately, the addition of monomers to this $\mathrm{CA}_{3}$ isomer results in low-symmetry structures that are unlikely building blocks for simple crystal forms. Moreover, these structures usually include one or 


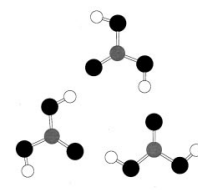

(a)

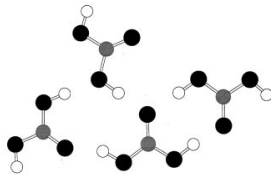

(b)

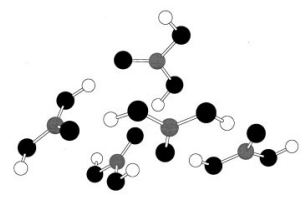

(c)

FIG. 7. Nonlinear isomers of carbonic acid clusters: (a) planar trimer, (b) planar tetramer, (c) a three-dimensional structure.

more dimers, suggesting that it may in fact be the most stable building block, not only in the linear clusters.

To investigate the possibility of bridging different linear chains by additional CA molecules, we have relaxed the system obtained by adding a single $\mathrm{CA}$ monomer close to the center of a linear $\mathrm{CA}_{5}$ cluster. A weak bond forms between a monomer hydrogen and an oxygen atom in the $\mathrm{CA}_{5}$ ladder, which remains stable and almost undeformed. The resulting three-dimensional geometry is a possible structural element for linking linear $\mathrm{CA}_{n}$ segments, but the cohesive energy of this bond is only $4.5 \mathrm{kcal} / \mathrm{mol}$, and it is probably less important than the van der Waals contribution. Other 3D structures generated by Car-Parrinello simulation or by performing DF calculations on structures produced by the model potential had cohesive energies well above those of the linear structures, and none had a symmetry suggesting the 3D crystals. An example of a 3D structure is shown in Fig. 7(c).

\section{DISCUSSION AND CONCLUDING REMARKS}

We have carried out an extensive series of density functional calculations on clusters of carbonic acid $\mathrm{H}_{2} \mathrm{CO}_{3}$ with up to five structural units. The calculations extend our earlier work on the monomer and other fragments of polycarbonate, ${ }^{9}$ and they appear to be the first calculations for clusters larger than the dimer. ${ }^{8}$ In addition to the geometrical structures, we calculate vibration frequencies and the energy cost of bending and stretching the clusters.

The most stable isomers are hydrogen-bonded, planar chains that follow the pattern found in the dimer. This is similar to that found in crystalline formic acid, ${ }^{28,29}$ where long chains of molecules linked by hydrogen bonds are held together by van der Waals forces. We know of no experimental evidence for the existence of such long chains in carbonic acid, which is a transient species in most environments, but we have shown that the chain structure is both stable against bending (a structure with $\sim 20 \mathrm{H}_{2} \mathrm{CO}_{3}$ units is needed before a ring becomes more stable than a chain) and has a substantial tensile strength. It would be most interest- ing to see whether such chain structures can be prepared. The structures of the carbonic acid polymorphs remains a challenge to theory and experiment.

\section{ACKNOWLEDGMENTS}

We thank the Forschungszentrum Jülich and the John von Neumann Institute for Computing (NIC) for providing generous access to the Cray T3E computers in the Forschungszentrum Jülich.

${ }^{1}$ See, for example, C. A. Wright and A. I. Boldyrev, J. Phys. Chem. 99, 12125 (1995), and references therein.

${ }^{2}$ K. R. Liedl, S. Sekušak, and E. Mayer, J. Am. Chem. Soc. 119, 3782 (1997). The most extensive calculations use the coupled cluster method $[\operatorname{CCSD}(\mathrm{T})]$ with the $6-311++\mathrm{G}(3 \mathrm{df}, 3 \mathrm{pd})$ basis.

${ }^{3}$ M. T. Nguyen and T.-K. Ha, J. Am. Chem. Soc. 106, 599 (1984); M. T. Nguyen, A. F. Hegarty, and T.-K. Ha, J. Mol. Struct.: THEOCHEM 150, 319 (1987).

${ }^{4}$ J. K. Terlouw, C. B. Lebrilla, and H. Schwarz, Angew. Chem. 99, 352 (1987); Angew. Chem. Int. Ed. Engl. 26, 354 (1987).

${ }^{5}$ K. I. Peterson and W. Klemperer, J. Chem. Phys. 80, 2439 (1984).

${ }^{6}$ M. H. Moore and R. K. Khanna, Spectrochim. Acta 47A, 255 (1991).

${ }^{7}$ W. Hage, A. Hallbrucker, and E. Mayer, J. Mol. Struct. 408/409, 527 (1997).

${ }^{8}$ W. Hage, K. R. Liedl, A. Hallbrucker, and E. Mayer, Science 279, 1332 (1998).

${ }^{9}$ B. Montanari, P. Ballone, and R. O. Jones, Macromolecules 32, 3396 (1999).

${ }^{10}$ A. Borrmann, B. Montanari, and R. O. Jones, J. Chem. Phys. 106, 8545 (1996).

${ }^{11}$ B. Montanari and R. O. Jones, Chem. Phys. Lett. 272, 347 (1997).

${ }^{12}$ N. Troullier and J. M. Martins, Phys. Rev. B 43, 1993 (1991).

${ }^{13}$ J. P. Perdew, K. Burke, and M. Ernzerhof, Phys. Rev. Lett. 77, 3865 (1996).

${ }^{14}$ R. Car and M. Parrinello, Phys. Rev. Lett. 55, 2471 (1985).

${ }^{15}$ CPMD program version 3.0, J. Hutter et al., Max-Planck-Institut für Festkörperforschung and IBM Research 1990-99.

${ }^{16}$ F. Sim, A. St-Amant, I. Papai, and D. R. Salahub, J. Am. Chem. Soc. 114, 4391 (1992)

${ }^{17}$ Simulations of liquid water, for example, are described in M. E. Tuckerman, K. Laasonen, M. Sprik, and M. Parrinello, J. Phys.: Condens. Matter 6, A93 (1994), and references therein.

${ }^{18}$ D. R. Hamann, Phys. Rev. B 55, R10157 (1997)

${ }^{19}$ M. Ernzerhof and G. E. Scuseria, J. Chem. Phys. 110, 5029 (1999).

${ }^{20}$ ORAC version 3.0. P. Procacci, T. A. Darden, E. Paci, and M. Marchi, J. Comput. Chem. 18, 1848 (1997).

${ }^{21}$ H. Sun, S. J. Mumby, J. R. Maple, and A. T. Hagler, J. Phys. Chem. 99, 5873 (1999).

${ }^{22}$ These numbers are for cohesive energies without zero point energies. The corresponding formula with ZPE corrections is $E_{n}=18.2$ $+15.8(n-2) \mathrm{kcal} / \mathrm{mol}$.

${ }^{23}$ The magnitude of the dipole moment is $0.27 \mathrm{D}, 0.71 \mathrm{D}$, and $0.47 \mathrm{D}$ for $n=1,3,5$, respectively.

${ }^{24}$ See, for example, R. O. Jones, J. Chem. Phys. 110, 5189 (1999). For $C_{n}$ the value of $n$ where the energies of chain and ring cross depends on the approximation used for the exchange-correlation energy.

${ }^{25}$ See, for example, N. A. Campbell, Biology, Fourth Edition (Benjamin Cummings, Menlo Park, CA, 1996).

${ }^{26}$ P. H. Gammon, H. Kiefte, and M. J. Clouter, J. Phys. Chem. 87, 4025 (1983).

${ }^{27}$ The mechanical strength of solids is, of course, lower than that implied by the strength of the molecular interactions, due to the presence of extended defects.

${ }^{28}$ F. Holtzberg, B. Post, and I. Fankuchen, Acta Crystallogr. 6, 127 (1953) (x-ray structure determination of crystalline formic acid).

${ }^{29}$ R. C. Millikan and K. S. Pitzer, J. Am. Chem. Soc. 80, 3515 (1958) (infrared spectra of dimeric and crystalline formic acid). 